\title{
Establecimiento de Pinus taeda en la provincia de Corrientes (Argentina): efecto del herbicida metsulfurón-metil sobre el crecimiento y la sobrevivencia de los plantines
}

\author{
Pinus taeda establishment in the province of Corrientes (Argentina): \\ effects of the herbicide metsulfuron-metil in seedlings growth and survival
}

\author{
Ana Garau1*, Gustavo Meyer', Susana Filippini de Delfino² \\ *Autor de correspondencia: ${ }^{1}$ Universidad de Buenos Aires, Facultad de Agronomía, Cátedra de Dasonomía, \\ Av. San Martín 4453 (1417), Buenos Aires, Argentina, Tel.: 54-11-45248018, agarau@agro.uba.ar \\ 2 Universidad de Buenos Aires, Facultad de Agronomía, Cátedra de Estadística.
}

\begin{abstract}
SUMMARY
With the objective to evaluate the effect of the herbicide metsulfurón-metil on Pinus taeda (loblolly pine) plantations, survival, herbicide injury on pines and seedlings growth were analyzed in an experiment in Gobernador Virasoro (North of Corrientes province, Argentina). Three doses of metsulturón-metil (30,60 and $90 \mathrm{~g} / \mathrm{ha}$ ) and two application zones over seedlings (total coverage a top and in the base of the seedlings) were analyzed. The effect of glyphosate application and a treatment without weed control were also studied. Herbicides were applied 30 days after plantation. Height and diameter at root collar were evaluated at the beginning of the trial; 60, 120 and 180 days after application. Survival and sanity of the seedlings were evaluated on day 60. No phytotoxic effect was evident, being seedlings vigorous with an intense green colour. No differences in growth were related to location of herbicide over the pine. Herbicides application produced greater growth than weeded control. With doses of 60 $\mathrm{g} / \mathrm{ha}$ and $90 \mathrm{~g} / \mathrm{ha}$ of metsulfurón-metil pines seedlings showed greatest diameter and height, being those results similar to glyphosate treatment.
\end{abstract}

Key words: herbicides, metsulfuron, Pinus taeda, plantations establishment.

\section{RESUMEN}

Con el objetivo de evaluar el efecto del uso del herbicida metsulfurón-metil en plantaciones de Pinus taeda (pino taeda) se analizaron la sobrevivencia, el daño del herbicida sobre las plantas y el crecimiento de los plantines en un ensayo realizado en Gobernador Virasoro (norte de la Provincia de Corrientes, Argentina). Se analizaron tres dosis de metsulfurón-metil (30, 60 y 90 $\mathrm{g} / \mathrm{ha}$ ) y dos zonas de aplicación del herbicida sobre los plantines forestales (cobertura total sobre la copa y dirigido a la base de la planta). Además, se evaluó la aplicación de glifosato y un tratamiento sin control de malezas. Los herbicidas se aplicaron 30 días después de la plantación. La altura y el diámetro a nivel del cuello se evaluaron al inicio del ensayo, 60, 120 y 180 días después de aplicado el producto; la sobrevivencia y el estado sanitario de los pinos a los 60 días. No se observó efecto fitotóxico sobre el pino, mostrando las plantas aspecto vigoroso y color verde intenso. El lugar de localización del herbicida sobre la planta no produjo diferencias en crecimiento. La aplicación de herbicidas produjo mayor crecimiento que el control enmalezado. Con dosis de 60 y $90 \mathrm{~g} / \mathrm{ha}$ de metsulfurón-metil las plantas mostraron mayor diámetro y altura, siendo estos resultados similares al tratamiento con glifosato.

Palabras clave: herbicida, metsulfurón-metil, Pinus taeda, fitotoxicidad, efectos en el crecimiento.

\section{INTRODUCCIÓN}

El norte de la provincia de Corrientes (Argentina) presenta características ventajosas en materia forestal, tanto desde el punto de vista productivo como económico, y entre las especies de mayor crecimiento en la zona se encuentra Pinus taeda L. (pino taeda) (SAGPyA 1999, Ibañez et al. 2004). Los procedimientos de plantación, el control de malezas, la aplicación de fertilizantes y el manejo de las plagas son factores de suma importancia en las primeras etapas de implantación de un cultivo forestal (Neary et al. 1990, Nambiar y Sands 1993). Numerosos trabajos describen la importancia del control de malezas en forestaciones de coníferas (Davies 1987, Britt 1992, Duran y Reboratti 1995, Wagner et al. 1996). En plantaciones jóvenes de pino taeda los tratamientos de fertilización y control de malezas herbáceas promueven el rápido crecimiento del pino (Tiarks y Haywood 1986, Zutter et al. 1987, Neary et al. 1990, Yeiser 2000), mientras que en plantaciones de Pinus radiata un buen con- 
trol de malezas resulta esencial, además, para garantizar una alta sobrevivencia inicial y un crecimiento uniforme de los plantines (McLaren 1997).

En el noreste de Corrientes (Argentina) el uso de glifosato, atrazina y acetoclor con pantallas protectoras para los plantines forestales muestra que la respuesta en crecimiento de pino taeda al control de malezas es de gran magnitud (Pezzutti 2000). Si bien el control químico es en la actualidad una buena alternativa para el manejo de las malezas, debe prestarse particular atención a la fitotoxicidad de los productos y controlar la deriva y/o la aplicación sobre las plantas forestales, ya que se pueden producir daños importantes o inclusive la muerte de los plantines (Durán y Reboratti 1995). La aplicación directa de ciertos herbicidas sobre las plantas forestales, dada su selectividad, representa una alternativa de menor costo económico, siendo necesario identificar herbicidas efectivos que amplíen el espectro de control o permitan extender el período de aplicación (Miller 1990).

Metsulfurón es un herbicida del grupo de las sulfonilureas utilizado en el control de varias especies de latifoliadas y algunas gramíneas anuales. Es fundamentalmente sistémico, absorbido tanto por follaje como por raíz, y es adecuadamente estable en mezclas de tanque con otros agroquímicos como clorsulfurón, 2,4-D, glifosato, difenzoquat y fertilizantes líquidos. Además, el producto comercial es degradado por la microbios naturales de los suelos y los compuestos resultantes son no-tóxicos y noherbicidas (Caseley 1996, CASAFE 2003). Presenta corta actividad residual y su adsorción está negativamente correlacionada con el $\mathrm{pH}$ del suelo y positivamente correlacionada con la materia orgánica del mismo; su solubilidad en agua aumenta de 60 ppm a pH 5 hasta 7.000 ppm a pH 7 (Caseley 1996, Sarmah et al. 1998, Pons y Barriuso 1999). Ensayos de fitotoxicidad de este producto sobre distintas especies de Pinus muestran resultados diversos. Dutkowski (1990) establece que en dosis de hasta $90 \mathrm{~g} / \mathrm{ha}$, aplicado un mes antes de la plantación de $P$. radiata causa ligeros daños y retraso en el crecimiento de los pinos taeda, mientras que aplicado 1 día antes de plantación causa mortandad con dosis de $10 \mathrm{~g} / \mathrm{ha}$. Smale (1989) no reporta efectos tóxicos con dosis de 150 o 300 g de metsulfurón aplicado un día o una semana antes de la plantación en la misma especie, si bien la mayor dosis produce menor crecimiento. Por su parte, plantines de $P$. resinosa presentan alta mortandad y plantines de $P$. banksiana síntomas de clorosis y ápices quemados por dosis de 35 a $142 \mathrm{~g} /$ ha de metsulfurón en aplicaciones de post-emergencia (Maass y Prouty 1985).

En el noreste de Corrientes y en la provincia de Misiones se está comenzando a utilizar el metsulfurón-metil en plantaciones de pinos (principalmente $P$. taeda y $P$. elliotii), pero no hay información en estas zonas que se refiera a la fitotoxicidad de distintas dosis o modos de aplicación de este herbicida.
El presente trabajo tuvo como objetivo establecer el efecto de la aplicación del herbicida metsulfurón-metil sobre la supervivencia, el estado sanitario y el crecimiento de plantines de $P$. taeda. Se analizaron tres dosis del herbicida y dos zonas de aplicación o localización del herbicida sobre los plantines de pino taeda (cobertura total sobre la copa o dirigido hacia la base con protección de la copa). Es importante poseer información sobre la factibilidad de uso de un herbicida que actúe en postemergencia de las malezas y que se pueda aplicar por encima del pino taeda, ya que permitiría un control de las malezas de manera más rápida, segura y económica.

\section{MÉTODOS}

El ensayo se realizó en el Establecimiento San Andrés, ubicado en las cercanías de Gobernador Virasoro, Corrientes, Argentina. El clima de la región es subtropical, húmedo, sin estación seca definida. Las precipitaciones anuales presentan un valor medio de $1.600 \mathrm{~mm}$ y la temperatura media anual es de $19,5^{\circ} \mathrm{C}$ (INTA 1990). Los suelos son rojos, arcillosos, profundos, ácidos ( $\mathrm{pH}$ $4,5)$ y con buena provisión de materia orgánica $(3 \%)$, correspondiendo al subgrupo kandiudalfes típico (INTA 1990). La comunidad vegetal en el lote de ensayo estaba constituida principalmente por Manihot flabellifolia, Euphorbia heterophylla, Maranta sp., Pfaffia tuberosa, Senna obtusifolia, Setaria poiretiana, Tragia uberabana, Eryngium ebracteatum, Paspalum plicatulum y Digitaria insularis.

Los plantines de $P$. taeda fueron producidos en bandejas rígidas de $93 \mathrm{~cm}^{3}$ (volumen/unidad). La implantación se realizó sobre taipas o camellones para favorecer el drenaje del lote y la aeración del suelo en donde se instaló la plantación. La plantación se efectuó en la primera quincena de julio y la distancia fue de 1,75 m entre los árboles en la línea y de 4 m entre las líneas (densidad 1.428 plantas/ha). Se realizó control químico de hormigas previo a la plantación y en los primeros meses del cultivo según el nivel de infestación. Se fertilizó con superfosfato triple $(200 \mathrm{~kg} / \mathrm{ha})$ y la aplicación e incorporación del fertilizante se realizó junto con la confección de la taipa.

Se probaron distintas dosis y zonas de aplicación o localización sobre los plantines del herbicida metsulfurón metil (Errasin ${ }^{\circledR}$, polvo mojable al $60 \%$, Chemotecnica), junto con un control enmalezado y un control limpio (con glifosato). Los niveles probados en el ensayo fueron los siguientes:

1. $30 \mathrm{~g} / \mathrm{ha}$ de metsulfurón metil aplicado en cobertura total sobre la copa del árbol.

2. $60 \mathrm{~g} / \mathrm{ha}$ de metsulfurón metil aplicado en cobertura total sobre la copa del árbol.

3. $90 \mathrm{~g} / \mathrm{ha}$ de metsulfurón metil aplicado en cobertura total sobre la copa del árbol. 
4. $30 \mathrm{~g} / \mathrm{ha}$ de metsulfurón metil con aplicación dirigida a la base del árbol.

5. $60 \mathrm{~g} / \mathrm{ha}$ de metsulfurón metil con aplicación dirigida a la base del árbol.

6. $90 \mathrm{~g} / \mathrm{h}$ de metsulfurón metil con aplicación dirigida a la base del árbol.

7. Control limpio con glifosato $(3 \%)$ con aplicación dirigida a la base del árbol.

8. Control enmalezado.

La aplicación de ambos herbicidas se realizó 30 días después de la plantación en franjas de 1,5 m. de ancho sobre la línea de plantación. Se utilizó una mochila de bombeo manual, de $22 \mathrm{~L}$ con un pico 8002, una presión de $2,8 \mathrm{~kg} / \mathrm{cm}^{2}$ y un volumen de $140 \mathrm{Ll}$ agua/ha. Se protegieron los plantines con bolsas de polietileno en el caso de las aplicaciónes de glifosato y metsulfurón-metil y las aplicaciones fueron dirigidas a la base de los mismos.

El ensayo fue realizado en bloques al azar con 6 repeticiones. Cada parcela estuvo compuesta por 12 plantas y sobre 8 de ellas se tomaron los valores de comportamiento. Las evaluaciones sobre los plantines forestales fueron: altura, diámetro a nivel del cuello y estado del árbol. La altura fue medida con una regla graduada (precisión $1 \mathrm{~mm}$ ), el diámetro con un calibre digital (Digimatic Calliper; precisión 0,01 mm) y el estado de los árboles se evaluó en forma cualitativa según una escala visual: 1, árbol vigoroso, color verde intenso; 2 , árbol falto de vigor, color verde claro; 3 , árbol débil, clorosis y necrosis; 4, árbol muy débil, muriendo; 5, árbol muerto. Las plantas se midieron el día de la aplicación del herbicida (Ti), a los 60 (T60), 120 (T120) y 180 (T180 = Tf) días de aplicado el producto. Se calculó el crecimiento durante los distintos períodos de evaluación (T60 - Ti; T120 Ti; T180 - Ti). La sobrevivencia y estado de los árboles se evaluaron 60 días luego de aplicado el producto.

Se realizó un análisis de variancia combinando los tratamientos con un enfoque factorial de dosis y lugar de localización del herbicida más un control enmalezado y un control limpio como tratamientos adicionales (Calzada Benza 1970), considerando un esquema de ensayo repetido en el tiempo (4 niveles) para las variables dependientes altura y diámetro. Dado que la prueba de esfericidad (Mauchly's) no permitió un enfoque univariado en el tiempo se probaron las hipótesis de igualdad de tratamientos siguiendo el enfoque multivariado (modelo lineal general para ensayos repetidos) analizando el efecto intratratamientos y entre tratamientos (Potvin et al. 1990, Kuehl 2000). Las comparaciones posteriores entre sujetos para el factor dosis y lugar se hicieron según las pruebas de Tukey y Ryan-Einot-Gabriel-Welsch (Kuehl 2000).

Posteriormente, y al observarse en los resultados del análisis estadístico que no hubo diferencias entre zonas de localización del herbicida sobre los plantines de pino taeda (cobertura total sobre la copa o dirigido a la base), se reordenaron los tratamientos para su análisis final: tratamiento 1: $30 \mathrm{~g}$ de metsulfurón; tratamiento 2: $60 \mathrm{~g}$ de metsulfurón; tratamiento 3: $90 \mathrm{~g}$ de metsulfurón; tratamiento 4: glifosato y tratamiento 5: testigo enmalezado. Se empleó el paquete estadístico SPSS (Statistical Product and Service Solutions) versión 11.5.

\section{RESULTADOS}

Supervivencia y fototoxicidad. La supervivencia resultó elevada $(97 \%)$. Los resultados mostraron que no hubo diferencias para los factores dosis $(P=0,33)$ y lugar de localización del herbicida sobre los plantines $(P=0,92)$ sobre el estado y supervivencia de las plantas de pino taeda (cuadro 1). De acuerdo a la escala de evaluación el estado de las plantas tratadas con metsulfurón presentó un valor medio de 1,34 $\pm 0,07$, lo que representa plantas vigorosas de color verde intenso.

Tamaño inicial (Ti). No se observaron diferencias en el tamaño inicial de las plantas asignadas a cada tratamiento. La altura media fue de $21,3 \pm 1,35 \mathrm{~cm}$, mientras que el diámetro a nivel del cuello fue de 2,85 \pm 0,52 $\mathrm{mm}$.

Diámetro final (DTf). En el análisis de los contrastes de los tratamientos en el tiempo y en el realizado entre tratamientos pudo observarse un efecto significativo de los distintos tratamientos de control de malezas sobre el diámetro de las plantas de pino taeda $(P<0,001)$ (figura 1$)$. El testigo enmalezado presentó el menor diámetro y los mayores valores estuvieron en el tratamiento controlado

Cuadro 1. Estado sanitario de los pinos taeda a los 60 días de la aplicación del herbicida metsulfurón metil en distintas dosis (30, 60 y $90 \mathrm{~g} / \mathrm{ha}$ ) y con dos localizaciones sobre las plantas (cobertura total sobre la copa y sobre la base).

Sanity stage of pines 60 days after metsulfuron methyl application in different doses $(30,60$ and $90 \mathrm{~g} / \mathrm{ha}$ ) and two locations over pine seedlings (total coverage a top and in the seedling base). References: 1: vigorous seedling, intense green colour; 2 without vigour, light green colour; 3 weak, chlorosis and necrosis; 4 too weak, dying; 5 dead.

\begin{tabular}{lr}
\hline \multicolumn{1}{c}{ Tratamientos } & Estado \\
\hline Tratamientos & \\
$30 \mathrm{~g} / \mathrm{ha}$ metsulfurón & 1,38 \\
$60 \mathrm{~g} / \mathrm{ha}$ metsulfurón & 1,24 \\
$90 \mathrm{~g} / \mathrm{ha}$ metsulfurón & 1,33 \\
glifosato & 1,10 \\
Enmalezado & 1,33 \\
Localización de la aplicación & \\
Cobertura total & 1,32 \\
Dirigido a la base & 1,36 \\
\hline
\end{tabular}


con glifosato y en el que recibió aplicación de 90 g de metsulfurón. Los tratamientos con 30 y $60 \mathrm{~g} / \mathrm{ha}$ de metsulfurón produjeron plantas con mayor diámetro que el testigo enmalezado, siendo las de $30 \mathrm{~g} / \mathrm{ha}$ significativamente menores que las que recibieron dosis de $90 \mathrm{~g} / \mathrm{ha}$.

Altura final (ATf). En el análisis de los contrastes de los tratamientos en el tiempo y en el realizado entre tratamientos pudo observarse un efecto significativo de los distintos tratamientos de control de malezas sobre la altura de las plantas de pino taeda $(P<0,001)$ (figura 2 ).

El testigo enmalezado presentó la menor altura final y no tuvo diferencias con el tratamiento de $30 \mathrm{~g}$ de metsulfurón. Las mayores alturas correspondieron a las aplicaciones de 60 g y 90 g de metsulfurón. Si bien no existió diferencia significativa entre el tratamiento con $30 \mathrm{~g}$ de metsulfurón y el de glifosato, este último produjo plantas más altas que el testigo enmalezado.

Crecimiento en diámetro y altura en el tiempo. Diámetro: en la evaluación realizada a los 60 días no se presentaron diferencias significativas $(P=0,190)$, pero sí las hubo a los $120(P<0,001)$ y 180 días $(P<0,001)$. A partir de los 120 días (T120) se observó que las plantas sin control de malezas presentaron menor diámetro que las tratadas con herbicidas; las plantas que recibieron glifosato mostraron mayor crecimiento que las tratadas

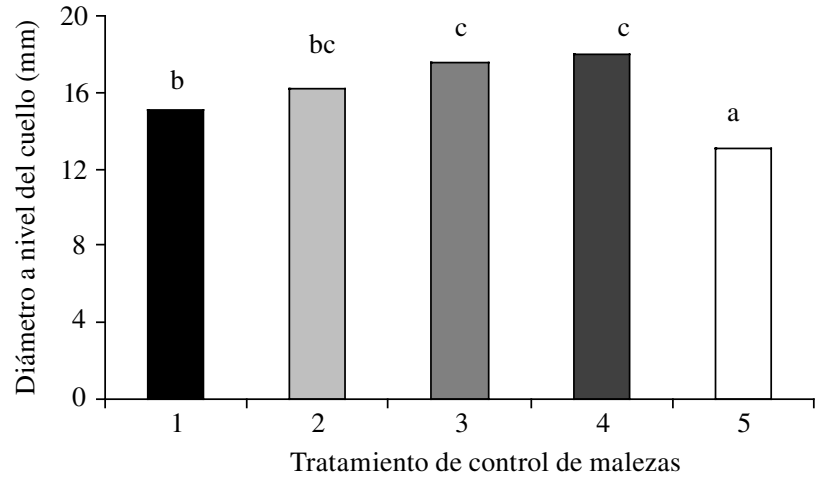

Figura 1. Diámetro a nivel del cuello en plantas de pino taeda de 11 meses con distintos tratamientos de control de malezas. Letras distintas indican diferencias significativas $(P<0,05)$. Referencias: 1: $30 \mathrm{~g}$ de metsulfurón; 2: $60 \mathrm{~g}$ de metsulfurón; 3: 90 g de metsulfurón; 4: glifosato; 5: testigo enmalezado.

Sanity stage of pines 60 days after metsulfuron methyl application in different doses (30,60 and $90 \mathrm{~g} / \mathrm{ha}$ ) and two locations over pine seedlings (total coverage a top and in the seedling base). References: 1: vigorous seedling, intense green colour; 2 without vigour, light green colour; 3 weak, chlorosis and necrosis; 4 too weak, dying; 5 dead.

con metsulfurón. A los 180 días (T180) el crecimiento en diámetro de las plantas con 60 y 90 g de metsulfurón resultó similar al obtenido en las plantas tratadas con glifosato (cuadro 2).

Cuadro 2. Crecimiento en diámetro y altura durante distintos períodos de evaluación para plantas de pino taeda con distintos tratamientos de control de malezas. Para cada período, letras distintas indican diferencias significativas $(P<0,05)$. En paréntesis la desviación estándar.

Diameter and height growth during different evaluation periods in pine seedlings with different weed control treatments. In each period different letters indicate a significant difference $(P<0.05)$.

\begin{tabular}{|c|c|c|c|}
\hline Período & Tratamiento $^{1}$ & Diámetro (mm) & Altura $(\mathrm{cm})$ \\
\hline \multirow[t]{5}{*}{60 días postaplicación } & 1 (30 g Metsulf) & $2,30(0,08) \mathrm{a}$ & $10,78(0,51) \mathrm{a}$ \\
\hline & $2(60 \mathrm{~g}$ Metsulf $)$ & $2,37(0,08) \mathrm{a}$ & $10,35(0,51) \mathrm{a}$ \\
\hline & 3 (90 g Metsulf) & $2,52(0,08) \mathrm{a}$ & $11,76(0,50) \mathrm{a}$ \\
\hline & 4 (glifosato) & $2,44(0,11) \mathrm{a}$ & $10,37(0,72) \mathrm{a}$ \\
\hline & 5 (enmalezado) & $2,26(0,12) \mathrm{a}$ & $10,14(0,71) \mathrm{a}$ \\
\hline \multirow[t]{5}{*}{120 días postaplicación } & 1 (30 g Metsulf) & $5,29(0,18) b$ & $26,38(0,95) b$ \\
\hline & 2 (60 g Metsulf) & $5,76(0,17) b$ & $26,94(0,94) b$ \\
\hline & 3 (90 g Metsulf) & $5,87(0,17) b$ & $26,35(0,94) b$ \\
\hline & 4 (glifosato) & $6,37(0,25) \mathrm{c}$ & $25,18(1,30) a b$ \\
\hline & 5 (enmalezado) & $4,29(0,25) \mathrm{a}$ & $21,48(1,33) \mathrm{a}$ \\
\hline \multirow[t]{5}{*}{180 días postaplicación } & 1 (30 g Metsulf) & $12,08(0,33) b$ & $44,33(1,34) b$ \\
\hline & 2 (60 Metsulf) & $13,07(0,33) b c$ & $47,81(1,34) b$ \\
\hline & 3 (90 g Metsulf) & $13,49(0,29) \mathrm{bc}$ & $48,93(1,34) b$ \\
\hline & 4 (glifosato) & $14,24(0,37) \mathrm{c}$ & $47,54(1,89) \mathrm{b}$ \\
\hline & 5 (enmalezado) & $9,34(0,37) \mathrm{a}$ & $32,73(1,79) \mathrm{a}$ \\
\hline
\end{tabular}

\footnotetext{
${ }^{1}$ Metsulf: Metsulfurón metil.
} 


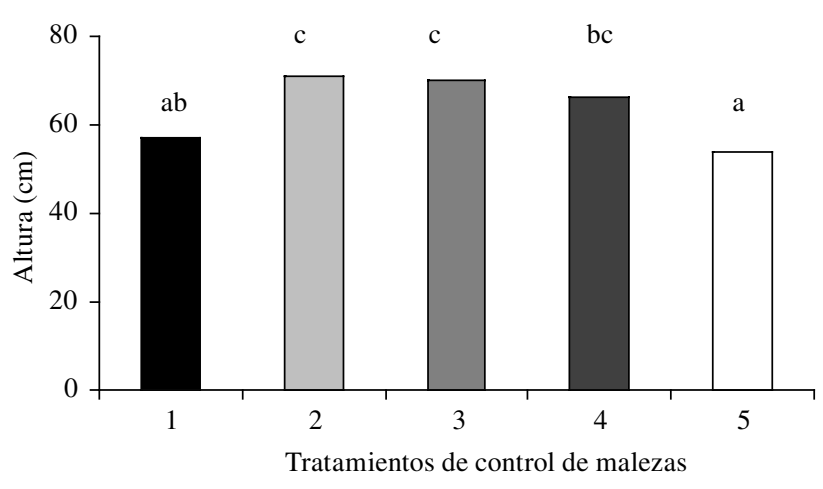

Figura 2. Altura en plantas de pino taeda de 11 meses con distintos tratamientos de control de malezas. Letras distintas indican diferencias significativas $(P<0,05)$. Referencias: 1: 30 g de metsulfurón; 2: $60 \mathrm{~g}$ de metsulfurón; 3: 90 g de metsulfurón; 4: glifosato; 5: testigo enmalezado.

Diameter and height growth during different evaluation periods in pine seedlings with different weed control treatments. In each period different letters indicate a significant difference $(P<0.05)$.

Cuadro 3. Diámetro a nivel del cuello de plantines de pino taeda 60, 120 y 180 días posteriores a la aplicación de metsulfurón metil en cobertura total sobre la copa del pino o sobre la base.

Root collar diameter in pine seedlings 60, 120 and 180 days after metsulfuron methyl in total coverage a top application and directed to the seedling base.

\begin{tabular}{ccc}
\hline $\begin{array}{c}\text { Días después de } \\
\text { la aplicación }\end{array}$ & $\begin{array}{c}\text { Localización de } \\
\text { la aplicación }\end{array}$ & $\begin{array}{c}\text { Diámetro } \\
(\mathrm{mm})\end{array}$ \\
\hline 60 & Cobertura total & 2,5 \\
120 & En la base & 2,2 \\
& Cobertura total & 5,5 \\
180 & En la base & 5,8 \\
& En la base & 12,7 \\
\hline
\end{tabular}

Altura. No se presentaron diferencias significativas $(P=0,260)$ a los 60 días, pero sí las hubo a los 120 $(P<0,01)$ y 180 días $(P<0,001)$. Las plantas sin control de malezas resultaron menores a los 120 días que las que recibieron aplicación de metsulfurón metil. A los 180 días no hubo diferencias entre tipo o dosis de herbicida, siendo estas plantas significativamente más altas que en el control enmalezado (cuadro 2).

Con respecto al lugar de localización de la aplicación de metsulfurón metil sobre los árboles, sólo se observó una tendencia $(P=0,10)$ a los 60 días y las plantas con aplicación de metsulfurón en la base presentaron mayor crecimiento en diámetro que las que recibieron la aplica- ción en la copa (2,5 mm contra 2,2 $\mathrm{mm}$ respectivamente). A los 120 y 180 días no se observaron diferencias ( $P=0,227$ y $P=0,291$ respectivamente) (cuadro 3 ). No hubo diferencias en el crecimiento en altura para ninguno de los períodos evaluados $(P=0,760, P=0,695$ y $P=0,995$ para 60,120 y 180 días respectivamente).

\section{DISCUSIÓN}

La aplicación de metsulfurón metil no presentó efecto de fitotoxicidad en ninguna de las dosis o lugares de aplicación sobre las plantas, siendo efectiva para el control de malezas en la plantación. González y Evans (1986) establecen que el metsulfurón metil es un herbicida seguro para Pinus sp. Smale (1989) y Dutkowski (1990) no reportan daños (o bien, citan daños muy leves) con aplicación preplantación de 150-300 g/ha o postplantación de $18 \mathrm{~g} /$ ha de metsulfurón respectivamente. Por su parte, Miller (1990) no cita mortandad en plantas de $P$. taeda de 3-4 años después de aplicar metsulfurón metil en dosis de 50 g/ha. Resulta importante destacar el contraste de estos resultados con los efectos de la aplicación de glifosato, ya que exponer los plantines forestales en su totalidad a ese herbicida produce graves daños en $P$. taeda (Miller 1990) y $P$. radiata (Gous 1997). Sin embargo, otros autores citan diversos síntomas de daño (clorosis, daño en yemas terminales) o incluso mortandad al utilizar metsulfurón metil en Pinus sp. Por ejemplo, el agregado de coadyuvantes como el siloxan junto con metsulfurón pueden provocar serios daños y muerte de plantas incluso con bajas dosis de metsulfurón (Dutkowski 1990). También influiría en su fitotoxicidad el momento de aplicación, puesto que Dutkowski (1990) reporta daños severos y mortandad en aplicaciones realizadas un día antes de la plantación, mientras que realizadas uno o dos meses antes de la misma no se producen daños.

Con respecto al lugar de localización del herbicida sobre la planta González y Evans (1986) establecen que la tolerancia de $P$. taeda al metsulfurón metil es alta y que la aplicación directa sobre el follaje es segura. Resultados similares reportan Miller (1990), Yeiser (2000) y Wood y Yeiser (2001); sin embargo, Michael y Andrews (1986) informan ligeros daños sobre el follaje en plantines de esa misma especie. Por su parte, Gous (1997) encuentra clorosis y muerte de yemas apicales en aplicaciones sobre la copa en $P$. radiata. Estos resultados contrastantes pueden adjudicarse a una distinta sensibilidad o tolerancia de las distintas especies de Pinus al efecto del herbicida. En tal sentido, Pinus resinosa y Pinus banksiana muestran claramente distinta tolerancia al metsulfurón, siendo el primero muy intolerante (Maas y Prouty 1985).

El lugar de aplicación del herbicida sobre la planta tampoco afectó el crecimiento, si bien en una única 
evaluación (crecimiento en diámetro a los 60 días de aplicado el herbicida) se observó una tendencia hacia un mayor crecimiento en las plantas con aplicaciones en la base. Este efecto temporal parcial sería similar al citado por Miller (1990), quien encuentra mayor crecimiento de diámetro en el mes de mayo con aplicaciones de $47 \mathrm{~g} / \mathrm{ha}$ de metsulfurón dirigidos hacia la base en $P$. taeda, mientras que en las evaluciones de julio y septiembre no se observan diferencias.

Las plantas tratadas con metsulfurón tuvieron mayor crecimiento que los controles enmalezados y las dosis de 60 y $90 \mathrm{~g} / \mathrm{ha}$ fueron las que permitieron los mayores diámetros finales y estas mismas dosis, junto con el tratamiento de uso glifosato, tuvieron la mayor altura final. Michael (1985), Yeiser (2000) y Wood y Yeiser (2001) también obtienen mayor crecimiento en plantas de $P$. taeda tratadas con metsulfurón. Sin embargo, estos resultados contrastan con lo reportado por Miller (1990) para $P$. taeda y por Dutkowski (1990) para $P$. radiata, quienes citan disminución en el crecimiento en altura después del tratamiento con metsulfurón metil, inclusive en dosis menores a $20 \mathrm{~g} / \mathrm{ha}$. No siempre se observan respuestas en crecimiento frente a la eliminación de la competencia por malezas. Michael (1985), Campbell (1987) y Quicke et al. (1996) reportan similar altura en plantas de pino taeda con y sin malezas, pero encuentran mayor crecimiento en diámetro (Michael 1985, Campbe11 1987). La menor sensibilidad de la altura ante la competencia que el diámetro puede explicarse a partir de la prioridad diferencial entre el crecimiento apical y el crecimiento del leño y a la alocación particular en condiciones de limitación de recursos (Waring y Schlesinger 1985, Kozlowski et al. 1991).

Para un manejo adecuado de las malezas durante la implantación es importante poder contar con un herbicida como el metsulfurón metil que actúe en postemergencia y que se pueda aplicar por encima del pino taeda, puesto que permitirá un control de las malezas de manera más rápida, segura y económica. Si bien las dosis probadas en este ensayo no muestran efectos dañinos sobre las plantas de pino taeda, como así tampoco la aplicación en cobertura directa sobre el follaje, nuevos experimentos son necesarios para generar información que amplíe el conocimiento referente al uso de este herbicida en plantaciones forestales.

\section{CONCLUSIONES}

Las dosis utilizadas (30, 60 y $90 \mathrm{~g} / \mathrm{ha}$ ) no produjeron efectos fitotóxicos en las plantas de pino taeda.

No se registraron daño o disminución de crecimiento en relación al lugar de localización del herbicida sobre las plantas (en cobertura total sobre la copa o aplicación dirigida hacia la base con la copa protegida).
A partir de los 120 días de la aplicación del herbicida se registró mayor crecimiento en las parcelas con metsulfurón que en las parcelas enmalezadas.

La aplicación de dosis de $90 \mathrm{~g} / \mathrm{ha}$ de metsulfurón produjo mayor altura y diámetro en las plantas de pino taeda de 11 meses que la dosis de $30 \mathrm{~g} / \mathrm{ha}$.

\section{REFERENCIAS}

Britt C. 1992.Residual herbicides for newly planted farm woodlands: Efficacy and tree tolerance. Aspects of Applied Biology, Vegetation management in forestry, amenity and conservation areas 29:211-218.

Calzada Benza J. 1970. Métodos estadísticos para la investigación. Lima, Perú. De Jurídica, $3^{\mathrm{a}}$ ed. 643 p.

CASAFE (Cámara Argentina de Sanidad Agropecuaria y Fertilizantes, AR.). 2003. Guía de Productos Fitosanitarios para la República Argentina. Tomo I. 752 p.

Campbell T. 1987. Pine growth response to chemical release from woody competition. Proceedings of the $40^{\text {th }}$ annual meeting of the Southern Weed Science Society. p. 249-254.

Caseley J. 1996. Herbicidas. In Labrada R, J Caseley, C Parker eds. Manejo de malezas para países en desarrollo. Estudio FAO-Producción y Protección Vegetal-120. FAO, Roma. p. 195-240.

Davies R. 1987. Trees and weeds. Weed control for successful tree establishment. Forestry Commission Handbook 2. London. HMSO. 36 p.

Durán M, H Reboratti. 1995. Alternativas de plantaciones forestales en el NE de Corrientes. Actas X Jornadas Forestales de Entre Ríos. p. 1-18.

Dutkowski G. 1990. Phytotoxicity of sulphonyl-urea herbicides to radiata pine. Proceedings of the $9^{\text {th }}$ Australian Weeds Conference. p. 530-534.

González F, R Evans. 1986. Escort herbicide foliar directed spray for pine release. Proceedings of the $39^{\text {th }}$ annual meeting of the Southern Weed Science Society. p. 240-245.

Gous S. 1997. Season of application affects herbicide efficacy in Pinus radiata plantations in the Souther Cape Region of South Africa. Southern African Forestry Journal 179:1-5.

Ibañez C, P Nuñez, R Pezzutti, F Rodríguez. 2004. Efectos de la roturación del suelo y fertilización con fósforo en el crecimiento inicial de plantaciones de Pinus taeda en suelos rojos del Nordeste de la provincia de Corrientes, Argentina. Bosque 25 (2):69-76.

INTA (Instituto Nacional de Tecnología Agropecuaria, AR). 1990. Atlas de suelos de la República Argentina. Tomo I. Secretaría de Agricultura, Ganadería y Pesca. Proyecto PNUD Arg. 85/019. Centro de Investigaciones en Recursos Naturales. $731 \mathrm{p}$.

Kozlowski T, P Kramer, S Pallardy. 1991. The physiological ecology of woody plants. Nueva York, USA. Academic Press. 411 p.

Kuehl R. 2000. Design of experiments. Statistical principles of research design and analysis. New York, USA. Duxbury. Second Edition. Section 15, Repeated Measures Designs. p. $492-513$.

Maass D, R Prouty. 1985. Efficacy and timing of metsulfuron methyl in forestry in Maine. Proceedings of the $40^{\text {th }}$ an- 
nual meeting of the Northeastern Weed Science Society. p. 230-232.

McLaren P. 1997. Radiata pine grower's manual. Chemical weed control. New Zealand. FRI $\mathrm{N}^{\circ}$ 184:33-39.

Michael J. 1985. Growth of loblolly pine treated with hexazinone, sulfometuron methyl and metsulfuron methyl for herbaceous weed control. Southern Journal of Applied Forestry 9:20-26.

Michael J, G Andrews. 1986. Pine tolerance to several new herbicides.

Proceedings of the $39^{\text {th }}$ annual meeting of the Sothern Weed Science Society. p. 218-221.

Miller J. 1990. Directed foliar sprays of forestry herbicides for loblolly pine release. Southern Journal of Applied Forestry 4:199-206.

Nambiar E, R Sands. 1993. Competition for water and nutrients in forests. Canadian Journal of Forest Research 23:1955-1968.

Neary D, D Rockwood, N Comerford, B Swindel, T Cooksey. 1990. Importance of weed control, fertilization, irrigation and genetics in slash and loblolly pine early growth on poorly drained spodosols. Forest Ecology and Management 30:271-281.

Pezzutti R. 2000. Efecto del control de malezas en el crecimiento inicial de plantaciones de Pinus taeda del Noreste de Corrientes, Argentina. Silvoargentina. Avances en el establecimiento de plantaciones de Coníferas Subtropicales en el Mercosur. Corrientes, Argentina. 16 p.

Pons N, E Barriuso. 1999. Fate of metsulfuron-methyl in soils in relation to pedo-climatic conditions. Pesticide Management Science 53 (4):311-323.

Potvin C, M Lechowicz, S Tardiff. 1990. The Statistical analysis of ecophysiological response curves obtained from experiments involving repeated measures. Ecology 71(4):1389-1400.
Quicke H, D Lauer, G Glover. 1996. Growth responses following herbicide release of loblolly pine from competing hardwoods in the Virginia Piedmont. Southern Journal of Applied Forestry 20 (4):177-181.

SAGPyA (Secretaría de Agricultura, Ganadería, Pesca y Alimentación, AR). 1999. Argentina: oportunidades de inversión en bosques cultivados. 208 p.

Sarmah A, R Kookana, A Alston. 1998. Fate and behaviour of triasulfuron, metsulfuron-methyl and chlorsulfuron in the Australian soil environment: a review. Australian Journal of Agricultural Research 49 (5):775-790.

Smale P. 1989. Desirable planting delay following preplant spraying with Escort in Northland. New Zealand Forestry:24-25.

Tiarks A, J Haywood. 1986. Pinus taeda L. Response to fertilization, herbaceous plant control, and woody plant control. Forest Ecology and Management 14:103-112.

Wagner R, T Noland, G Mohammed. 1996. Timing and duration of herbaceous vegetation control around four northern coniferous species. New Zealand Journal of Forestry Science 26:39-52.

Waring R, W Schlesinger. 1985. Forest Ecosystems. Concepts and Management. New York, USA. Academic Press. $340 \mathrm{p}$.

Wood R, J Yeiser. 2001. Herbaceous weed control and resultant pine seedling growth with new Oust, Velpar and Escort formulations: year two results. Proceedings of the Southern Weed Science Society 54:109-113.

Yeiser J. 2000. New formulations of Oust, Velpar and Escort for herbaceous weed control. Proceedings of the Southern Weed Science Society 53:124-128.

Zutter B, G Glover, D Gjerstad. 1987. Vegetation response to intensity of herbaceous weed control in a newly planted loblolly pine plantation. New Forest 4:257-271. 